\title{
Choice and Outcomes of Rate Control versus Rhythm Control in Elderly Patients with Atrial Fibrillation: A Report from the REPOSI Study
}

\author{
Francesco Paciullo $^{1,2} \cdot$ Marco Proietti $^{3} \cdot$ Vanessa Bianconi $^{1}{ }^{1} \cdot$ Alessandro Nobili $^{4}$. \\ Matteo Pirro $^{1}$ - Pier Mannuccio Mannucci ${ }^{5}$ Gregory Y. H. Lip ${ }^{3,6}$ • \\ Graziana Lupattelli ${ }^{1} \cdot$ REPOSI Investigators
}

Published online: 21 March 2018

(C) Springer International Publishing AG, part of Springer Nature 2018

\begin{abstract}
Background Among rate-control or rhythm-control strategies, there is conflicting evidence as to which is the best management approach for non-valvular atrial fibrillation (AF) in elderly patients.

Design We performed an ancillary analysis from the 'Registro Politerapie SIMI' study, enrolling elderly inpatients from internal medicine and geriatric wards.
\end{abstract}

Gregory Y.H. Lip and Graziana Lupattelli were joint senior authors on this work.

Francesco Paciullo and Marco Proietti contributed equally to this work.

The members of the REPOSI Investigators have been listed in Acknowledgements.

Electronic supplementary material The online version of this article (https://doi.org/10.1007/s40266-018-0532-8) contains supplementary material, which is available to authorized users.

Vanessa Bianconi

v.bianconi.vb@gmail.com

1 Unit of Internal Medicine, Department of Medicine, University of Perugia, Piazzale Menghini, Sant'Andrea delle Fratte, 06132 Perugia, Italy

2 Section of Internal and Cardiovascular Medicine, Department of Medicine, University of Perugia, Perugia, Italy

3 Institute of Cardiovascular Sciences, University of Birmingham, Birmingham, UK

4 Department of Neuroscience, IRCCS-Istituto di Ricerche Farmacologiche Mario Negri, Milan, Italy

5 Angelo Bianchi Bonomi Hemophilia and Thrombosis Center, IRCCS Fondazione Cà Granda, Milan, Italy
Methods We considered patients enrolled from 2008 to 2014 with an AF diagnosis at admission, treated with a rate-control-only or rhythm-control-only strategy.

Results Among 1114 patients, 241 (21.6\%) were managed with observation only and $122(11 \%)$ were managed with both the rate- and rhythm-control approaches. Of the remaining 751 patients, $626(83.4 \%)$ were managed with a rate-control-only strategy and $125(16.6 \%)$ were managed with a rhythm-control-only strategy. Rate-control-managed patients were older $(p=0.002)$, had a higher Short Blessed Test (SBT; $p=0.022$ ) and a lower Barthel Index $(p=0.047)$. Polypharmacy $(p=0.001)$, heart failure $(p=0.005)$ and diabetes $(p=0.016)$ were more prevalent among these patients. Median $\mathrm{CHA}_{2} \mathrm{DS}_{2}$-VASc score was higher among rate-control-managed patients $(p=0.001)$. SBT [odds ratio (OR) $0.97,95 \%$ confidence interval (CI) $0.94-1.00, p=0.037$ ], diabetes (OR $0.48,95 \%$ CI $0.26-0.87, p=0.016$ ) and polypharmacy (OR $0.58,95 \%$ CI $0.34-0.99, p=0.045)$ were negatively associated with a rhythm-control strategy. At follow-up, no difference was

6 Aalborg Thrombosis Research Unit, Department of Clinical Medicine, Aalborg University, Aalborg, Denmark 
found between rate- and rhythm-control strategies for cardiovascular (CV) and all-cause deaths (6.1 vs. 5.6\%, $p=0.89$; and 15.9 vs. $14.1 \%, p=0.70$, respectively).

Conclusion A rate-control strategy is the most widely used among elderly AF patients with multiple comorbidities and polypharmacy. No differences were evident in $\mathrm{CV}$ death and all-cause death at follow-up.

\section{Key Points}

Rate control is the preferred therapeutic choice in elderly atrial fibrillation (AF) patients with multiple comorbidities and polypharmacy.

No difference is evident between the rate- and rhythm-control strategies in cardiovascular death and all-cause death in elderly AF patients.

\section{Introduction}

Atrial fibrillation (AF) is a major public health burden and is the most common cardiac arrhythmia worldwide, with a major impact on morbidity and mortality [1-3]. The main goals of therapy in AF are the prevention of cardioembolic events with anticoagulation and the management of symptoms with a rhythm- or rate-control strategy [3-5].

Randomized clinical trials have compared the effectiveness of rhythm- and rate-control strategies. Rate control was non-inferior to rhythm control for several primary outcomes related to symptom management, as well as cardiac adverse events and overall mortality [6-9]. Rhythm control was also associated with a higher risk of rehospitalization [8, 9]. Furthermore, data from a large observational study showed no superiority for rhythm-control compared with a rate-control strategy in regard to outcomes such as stroke, heart failure or mortality, but rhythm control was associated with more cardiovascular (CV) hospitalizations [10]. On the other hand, observational studies on hospitalized AF patients suggest that rhythmcontrol strategies may have a marginal benefit on mortality compared with rate control during long-term follow-up [11]. In randomized trials, the mean age of patients was generally lower than 70 years, while in observational studies, the mean age of patients was approximately 75 years. Thus, limited data on the outcomes regarding rate- or rhythm-control strategies are available for elderly patients.
With these gaps of knowledge in elderly patients, we chose to compare clinical outcomes related to rate-control versus rhythm-control strategies for the management of $\mathrm{AF}$ in elderly patients admitted to internal medicine and geriatric wards in the frame of the prospective Registro Politerapie SIMI (REPOSI) registry.

\section{Methods}

This study represents an ancillary analysis from the REPOSI study [12], a multicentre collaborative observational registry jointly held by the Italian Society of Internal Medicine (SIMI), the Ca' Granda Maggiore Policlinico Hospital Foundation, and the Mario Negri Institute of Pharmacological Research, and based on a network of internal medicine and geriatric wards in Italy and Spain. Full details on study design and specific aims have been reported [12].

Briefly, REPOSI was conducted for 3 non-consecutive years $(2008,2010,2012)$ and then annually from 2014 onwards. In each of these years, consecutive patients admitted to the participating wards and aged $>65$ years were enrolled over a period of 4 weeks on a quarterly basis (i.e. February, June, September and December). The study protocol was first approved by the Ethics Committee of the Ca' Granda Maggiore Policlinico Hospital Foundation and then ratified for each enrolling site by the local committees. The study was conducted according to Good Clinical Practice recommendations and the Declaration of Helsinki. Concomitant diagnoses at admission were coded according to the International Classification of Diseases, 9th Revision (ICD-9) system. Medication use at admission was assessed according to the Anatomic Therapeutic Chemical (ATC) classification system.

For the purposes of this analysis, we included all patients admitted from 2008 to 2014 with a diagnosis of AF and treated with a rate- or rhythm-control management strategy. AF was classified according to ICD-9 code 427.31. A rate-control strategy was defined for patients treated with $\beta$-blockers (ATC $\mathrm{C} 07^{*}$ ), calcium-channel blockers (ATC $\mathrm{C} 08 \mathrm{C}^{*}$ ) or digoxin (ATC C01AA05), while rhythm control was defined for patients treated with class IC anti-arrhythmic drugs (ATC C01BC*) or class III antiarrhythmic drugs (ATC C01BD*). Adequate heart rate control was classified according to the prevailing European Society of Cardiology (ESC) guidelines, i.e. heart rate $<110$ beats per min (bpm) at admission [13].

Interactions of comorbidities were evaluated by means of the Cumulative Illness Rating Scale (CIRS) severity index and comorbidity index $[14,15]$. Polypharmacy was defined by the contemporary use of five or more drugs [12] Cognitive status was evaluated using the Short Blessed 
Test (SBT) [16], the presence of depression was investigated using a Geriatric Depression Scale (GDS) [17], and patient functional status was assessed using the Barthel index [18].

Follow-up data were collected at 3 and/or 12 months after discharge through a patient telephone interview, or, if the patient was no longer alive, from the next of kin. According to the causes of death reported in the electronic case report form, a CV death was classified as such when it was related to any cardiac or vascular reason. Both allcause and CV deaths were considered study outcomes.

\subsection{Statistical Analysis}

Variables with normal distribution were expressed as means and standard deviations (SDs), and tested for differences using the Student's $t$ test. Non-normal variables were expressed as medians and interquartile ranges (IQRs), and differences were tested using the Mann-Whitney $U$ test or Kruskall-Wallis analysis of variance (ANOVA) test accordingly. Categorical variables, expressed as counts and percentages, were analysed using the Chi-square test.

A logistic regression analysis was performed to establish clinical factors associated with rhythm-control management. All variables with a $p$ value $<0.10$ in the comparison between the two groups at baseline were included in a univariate analysis, and the univariate predictors with a statistical significance of $<10 \%$ were included in a multivariate logistic model. A linear regression analysis, adjusted for age, sex and CIRS, was performed to analyse the relationship between rhythm control and heart rate at admission.

A survival analysis was performed according to the rateand rhythm-control strategies. A log-rank test was only performed for the 'all-cause' death outcome owing to a low number of events for $\mathrm{CV}$ death. A two-sided $p$ value $<0.05$ was considered statistically significant. All analyses were performed using SPSS version 22.0 (IBM Corporation, Armonk, NY, USA).

\section{Results}

Of 1114 patients with $\mathrm{AF}$ at admission, 241 (21.6\%) were managed with observation only (neither rate- nor rhythmcontrol drugs were prescribed), and $122(11 \%)$ were managed with both rate- and rhythm-control drugs. Of the remaining 751 patients included in this analysis, 626 $(83.4 \%)$ were managed with rate-control drugs only, and $125(16.6 \%)$ were managed with rhythm-control drugs only.

Baseline characteristics at hospital admission for these 751 patients, according to rate- or rhythm-control strategies, are summarized in Table 1. Comparison of demographic and clinical characteristics between the two groups indicated that rate-control-managed patients were older ( $U$ test $32.112, p=0.002$ ) and had a higher SBT ( $U$ test $17.440, p=0.022$ ) and lower Barthel Index ( $U$ test 25.558, $p=0.047$ ). Polypharmacy (Chi-square test 10.846, $p=0.001$ ), heart failure (Chi-square test 7.858, $p=0.005$ ) and diabetes (Chi-square test 5.775, $p=0.016$ ) were more prevalent among these patients. Furthermore, median $\mathrm{CHA}_{2} \mathrm{DS}_{2}$-VASc score was higher among ratecontrol-managed patients ( $U$ test 34.031, $p=0.001$ ), but the proportion of those at high risk (score $\geq 2$ ) was not different between the two groups (Chi-square test 2.448, $p=0.12$ ). Patients managed with rate-control drugs were more likely prescribed oral anticoagulant (OAC) monotherapy than those managed with rhythm-control drugs, who, conversely, were more untreated and were prescribed antiplatelet monotherapy (Chi square test 7.906, $p=0.048)$.

\subsection{Logistic Regression Analysis for Rhythm Control}

After univariate analysis, a multivariate logistic analysis found that the SBT [odds ratio (OR) $0.97,95 \%$ confidence interval (CI) 0.94-1.00, $p=0.037$ ], diabetes (OR 0.48, 95\% CI $0.26-0.87, p=0.016$ ) and polypharmacy (OR $0.58,95 \%$ CI $0.34-0.99, p=0.045$ ) were inversely associated with the use of a rhythm-control strategy.

\subsection{Heart Rate Control}

Median heart rate at admission was significantly higher in patients managed with a rate-control strategy than in those managed with rhythm control [median (IQR) 80 (72-96) vs. 76 (66-88) bpm, respectively; $U$ test 30.558, $p<0.001]$, but no significant differences were found in the prevalence of adequate heart rate control between the two groups $(90.7 \%$ vs. $91.2 \%$, respectively; Chi-square test $0.036, p=0.85$ ). A rhythm-control strategy was negatively associated with a lower heart rate at admission, even after adjustment for age, sex and Cumulative Index Rating Scale (standardized $\beta=-0.128, t=-3.141, p=0.002$ ).

\subsection{Follow-Up Analysis}

Follow-up data were available for 418 of 751 (55.7\%) patients. Rates of CV death and all-cause deaths were not different between the rate- and rhythm-control groups (6.1 vs. $5.6 \%$; Chi-square test $0.018, p=0.89$, and 15.9 vs. $14.1 \%$; Chi-square test $0.140, p=0.70$, respectively). Kaplan-Meier analysis showed no significant differences 
Table 1 Baseline characteristics of patients according to the management strategy for atrial fibrillation

\begin{tabular}{|c|c|c|c|c|}
\hline & Rate control $[n=626]$ & Rhythm control $[n=125]$ & U/Chi-square test & $P$-Value \\
\hline Age, years (median [IQR]) & $82[76-86]$ & 80 [74-84] & $32.112^{\mathrm{a}}$ & 0.002 \\
\hline Female & $320(51.1)$ & $64(51.2)$ & $0.000^{\mathrm{b}}$ & 0.99 \\
\hline SBT (median [IQR]) 547 & $10[4-17]$ & $7[2-14]$ & $17.440^{\mathrm{a}}$ & 0.022 \\
\hline GDS (median [IQR]) 519 & $1[0-2]$ & $1[0-3]$ & $18.926^{\mathrm{a}}$ & 0.91 \\
\hline Barthel Index (median [IQR]) 584 & $87[53-100]$ & $91[69-100]$ & $25.558^{\mathrm{a}}$ & 0.047 \\
\hline \multicolumn{5}{|l|}{ CIRS (median [IQR]) 590} \\
\hline Severity Index & $1.69[1.53-1.94]$ & $1.69[1.46-1.98]$ & $22.575^{\mathrm{a}}$ & 0.46 \\
\hline Comorbidity Index & $3[2-5]$ & $3[2-5]$ & $22.351^{\mathrm{a}}$ & 0.37 \\
\hline Polypharmacy 546 & $508(81.2)$ & $85(68.0)$ & $10.846^{\mathrm{b}}$ & 0.001 \\
\hline Hypertension & $497(79.4)$ & $92(73.6)$ & $2.067^{\mathrm{b}}$ & 0.15 \\
\hline Hypercholesterolemia & $48(7.7)$ & $9(7.2)$ & $0.033^{\mathrm{b}}$ & 0.86 \\
\hline Heart failure & $193(30.8)$ & $23(18.4)$ & $7.858^{\mathrm{b}}$ & 0.005 \\
\hline Coronary artery disease & $167(26.7)$ & $25(20.0)$ & $2.441^{\mathrm{b}}$ & 0.12 \\
\hline Myocardial infarction & $20(3.2)$ & $2(1.6)$ & $0.932^{\mathrm{b}}$ & 0.33 \\
\hline Peripheral arterial disease & $23(3.7)$ & $3(2.4)$ & $0.506^{\mathrm{b}}$ & 0.48 \\
\hline Stroke/TIA & $78(12.5)$ & $16(12.8)$ & $0.011^{\mathrm{b}}$ & 0.92 \\
\hline Diabetes mellitus & $192(30.7)$ & $25(20.0)$ & $5.775^{\mathrm{b}}$ & 0.016 \\
\hline Chronic kidney disease & $160(25.6)$ & $26(20.8)$ & $1.267^{\mathrm{b}}$ & 0.26 \\
\hline COPD & $172(27.5)$ & 27 (21.6) & $1.847^{\mathrm{b}}$ & 0.17 \\
\hline Cancer & $72(11.5)$ & $21(16.8)$ & $2.696^{\mathrm{b}}$ & 0.101 \\
\hline $\mathrm{CHA}_{2} \mathrm{DS}_{2}-\mathrm{VASc}($ median $[\mathrm{IQR}])$ & $4[3-5]$ & $3[3-5]$ & $34.031^{\mathrm{a}}$ & 0.001 \\
\hline \multicolumn{5}{|l|}{ Thromboembolic risk } \\
\hline Moderate risk & $8(1.3)$ & $4(3.2)$ & $2.448^{\mathrm{b}}$ & 0.12 \\
\hline High risk & $618(98.7)$ & $121(96.8)$ & & \\
\hline \multicolumn{5}{|l|}{ Antithrombotic therapy } \\
\hline None & $133(21.2)$ & $33(26.4)$ & $7.906^{\mathrm{b}}$ & 0.048 \\
\hline Only antiplatelet & $189(30.2)$ & 47 (37.6) & & \\
\hline Only OAC & $282(45.0)$ & $44(35.2)$ & & \\
\hline Antiplatelet plus OAC & $22(3.5)$ & $1(0.8)$ & & \\
\hline
\end{tabular}

Data are expressed as $n(\%)$ unless otherwise specified

${ }^{a}$ Mann-Whitney $U$ test

${ }^{\mathrm{b}} \mathrm{Chi}$-square test

CIRS Cumulative Index Rating Scale, COPD chronic obstructive pulmonary disease, GDS Geriatric Depression Scale, IQR interquartile range, $O A C$ oral anticoagulant, SBT Short Blessed Test, TIA transient ischemic attack

between the two treatment groups (log-rank 0.002 , $p=0.96)$ for the occurrence of all-cause death.

\subsection{Sensitivity Analysis}

In order to deepen this analysis, we performed a sensitivity analysis comparing baseline characteristics of the ratecontrol- and rhythm-control-only groups with those managed with observation only and those managed with a composite rate- and rhythm-control strategy (Electronic supplementary Table S1).

Patients managed with observation only were found to be the oldest, while those managed with a composite strategy were the youngest (Kruskal-Wallis test 24.899, $p<0.001)$. Patients assigned to the observation-only strategy were cognitively impaired as much as those assigned to rate-control only, while patients treated with the combined strategy were those less likely to be cognitively impaired according to the SBT (Kruskal-Wallis test 35.065, $p<0.001)$. Even though there was a non-significant difference, patients assigned to the observation strategy were as much functionally impaired as those assigned to rate control, as evaluated by the Barthel Index, while those treated with both strategies were the most competent (Kruskal-Wallis test 7.493, $p=0.058$ ). Patients assigned to the observation-only strategy were less burdened with 
polypharmacy, while those assigned to both strategies were more burdened with polypharmacy (Chi-square test 79.349, $p<0.001$ ). Patients treated with a combined strategy were also more affected with hypertension and coronary artery disease.

\section{Discussion}

In this cohort of elderly AF patients, a rate-control strategy was the most widely used, especially among patients with multiple comorbidities and related polypharmacy. Conversely, patients on a rhythm-control strategy were healthier, with less comorbidities, less polypharmacy and a less compromised cognitive status. No difference was found in terms of major adverse outcomes between the two management strategies.

After several randomized trials found no difference between the rate-control and rhythm-control strategies for prognosis $[7,8,19]$, a significant reduction in the use of rhythm-control management was evident, with rate control being the more widely used strategy $[10,11,20,21]$. Our data not only confirm previous evidence but also show, in a large contemporary 'real-world' cohort, that rate control remains the preferred management in elderly patients, with $83 \%$ of our cases being managed with rate-control drugs.

The main indication for rhythm control is the management of symptoms [22], with no specific guidance in relation to the degree of frailty or advanced age. In the present study, the choice of rate control seems associated with functional status, with these patients being more frail, with more comorbidities, more medications, and a worse cognitive status. This choice is likely to be explained by the fact that the rate control may be perceived as being more conservative and less likely to burden these patients with side effects, more frequent follow-ups or hospitalizations [23, 24]. In addition, rate control has been associated with an improvement of health-related quality of life in elderly patients [25, 26]. Importantly, differential management strategies did not significantly affect outcomes during the short-term follow-up observation in our cohort, suggesting a more prominent role of comorbidities.

Our data in elderly patients with AF should be integrated in the context of more general data available from the literature. The role of either rate- or rhythm-control management in predicting long-term outcomes in AF is still largely debated. While randomized trials found no differences in outcomes $[7,8]$, some observational studies suggest a better prognosis for rhythm control [27, 28], while others report that the difference between rate and rhythm control is no longer significant after full adjustment for comorbidities [10]. In elderly patients, scarce data are available, with rhythm control being reported as associated with both better [29] and worse outcomes [30].

The further comparison between the rate-control-only and rhythm-control-only groups and those assigned to observation only, as well as those treated with both strategies, confirmed and extended the main results. Indeed, we found that patients treated with an observationonly approach were the oldest and were slightly more cognitively and functionally compromised than those assigned to rate-control only. Conversely, those patients treated with both strategies were younger and significantly more 'fit' than the others and were keen to receive a more aggressive approach, which is probably justified by the fact that these patients are significantly more burdened with $\mathrm{CV}$ comorbidities. The fact that observation-only patients are less burdened with polypharmacy could be related to their severely impaired clinical status, which did not allow several drugs to be prescribed.

The arguably 'more conservative' approach of the observation-only and rate-control-only strategies in very old and complex patients could be a reasonable choice given the possibly easier management approach, favoring symptom control and improvement in quality of life. Nonetheless, given the small sample size and the nature of the study, this study is intended to simply be hypothesesgenerating and larger studies would be needed to further verify our data and assumptions.

The main limitation of this study is its observational nature, with the likelihood of residual confounders that could not be accounted for. This subgroup analysis was not prespecified, therefore we had limited power to identify differences between the groups. Moreover, we were not able to consider other possible rhythm-control management procedures (i.e. direct cardioversion, ablation procedure). Indeed, data regarding the choice of rate control or rhythm control were indirectly based on drug prescription data collected at baseline. In addition, no specific data were available about type of AF and AF-related symptoms. Last, the limited follow-up data after hospital release could reduce the generalizability of our conclusions. Despite these limitations, we believe that these data provide a representative 'real-world' snapshot of rate- and rhythmcontrol management strategies in elderly patients with AF.

\section{Conclusions}

We found that in elderly AF patients, a rate-control strategy is the most widely used, especially among those with multiple concomitant illnesses and polypharmacy. No significant differences were evident in CV death and allcause death at follow-up between the rate- or rhythmcontrol strategies. A rate-control strategy could be 
considered as the best choice for elderly AF patients; however, further data are needed to confirm our hypotheses.

Acknowledgements Investigators and co-authors of the REPOSI (REgistro POliterapie SIMI, Società Italiana di Medicina Interna) Study Group.

Steering Committee: Pier Mannuccio Mannucci (Chair, Fondazione IRCCS Cà Granda Ospedale Maggiore Policlinico, Milano), Alessandro Nobili (co-chair, IRCCS-Istituto di Ricerche Farmacologiche "Mario Negri", Milano), Mauro Tettamanti, Luca Pasina, Carlotta Franchi (IRCCS-Istituto di Ricerche Farmacologiche "Mario Negri”, Milano), Francesco Perticone (Presidente SIMI), Francesco Salerno (IRCCS Policlinico San Donato Milanese, Milano), Salvatore Corrao (ARNAS Civico, Di Cristina, Benfratelli, DiBiMIS, Università di Palermo, Palermo), Alessandra Marengoni (Spedali Civili di Brescia, Brescia), Giuseppe Licata (Azienda Ospedaliera Universitaria Policlinico P. Giaccone di Palermo, Palermo, Medicina Interna e Cardioangiologia), Francesco Violi (Policlinico Umberto I, Roma, Prima Clinica Medica), Gino Roberto Corazza, (Reparto 11, IRCCS Policlinico San Matteo di Pavia, Pavia, Clinica Medica I), Maura Marcucci (Unità di Geriatria, Fondazione IRCCS Ca' Granda Ospedale Maggiore Policlinico \& Dipartimento di Scienze Cliniche e di Comunità, Università degli Studi di Milano, Milano, Italia).

Clincal data monitoring and revision: Tarek Kamal Eldin, Maria Pia Donatella Di Blanca, Giovanna Lanzo, Sarah Astuto (IRCCSIstituto di Ricerche Farmacologiche "Mario Negri", Milano).

Database Management and Statistics: Mauro Tettamanti, Ilaria Ardoino, Laura Cortesi (IRCCS-Istituto di Ricerche Farmacologiche "Mario Negri", Milano).

Investigators: Italian Hospitals: Domenico Prisco, Elena Silvestri, Caterina Cenci, Giacomo Emmi (Azienda Ospedaliero Universitaria Careggi Firenze, Medicina Interna Interdisciplinare); Gianni Biolo, Gianfranco Guarnieri, Michela Zanetti, Giovanni Fernandes, Massimiliano Chiuch (Azienda Ospedaliera Universitaria Ospedali Riuniti di Trieste, Trieste, Clinica Medica Generale e Terapia Medica); Massimo Vanoli, Giulia Grignani, Gianluca Casella, Edoardo Alessandro Pulixi (Azienda Ospedaliera della Provincia di Lecco, Ospedale di Merate, Lecco, Medicina Interna); Mauro Bernardi, Silvia Li Bassi, Luca Santi, Giacomo Zaccherini (Azienda Ospedaliera Policlinico Sant'Orsola-Malpighi, Bologna, Semeiotica Medica Bernardi); Elmo Mannarino, Graziana Lupattelli, Vanessa Bianconi, Francesco Paciullo (Azienda Ospedaliera Santa Maria della Misericordia, Perugia, Medicina Interna, Angiologia, Malattie da Arteriosclerosi); Ranuccio Nuti, Roberto Valenti, Martina Ruvio, Silvia Cappelli, Alberto Palazzuoli (Azienda Ospedaliera Università Senese, Siena, Medicina Interna I); Teresa Salvatore, Ferdinando Carlo Sasso (Azienda Ospedaliera Universitaria della Seconda Università degli Studi di Napoli, Napoli, Medicina Interna e Malattie Epato-Bilio Metaboliche Avanzate); Domenico Girelli, Oliviero Olivieri, Thomas Matteazzi (Azienda Ospedaliera Universitaria Integrata di Verona, Verona, Medicina Generale a indirizzo Immuno-Ematologico $e$ Emocoagulativo); Mario Barbagallo, Lidia Plances, Roberta Alcamo (Azienda Ospedaliera Universitaria Policlinico Giaccone Policlinico di Palermo, Palermo, Unità Operativa di Geriatria e Lungodegenza); Giuseppe Licata, Luigi Calvo, Maria Valenti (Azienda Ospedaliera Universitaria Policlinico P. Giaccone di Palermo, Palermo, Medicina Interna e Cardioangiologia); Marco Zoli, Raffaella Arnò (Azienda Ospedaliera Universitaria Policlinico S. Orsola-Malpighi, Bologna, Unità Operativa di Medicina Interna Zoli); Franco Laghi Pasini, Pier Leopoldo Capecchi, Maurizio Bicchi (Azienda Ospedaliera Universitaria Senese, Siena, Unità Operativa Complessa
Medicina 2); Giuseppe Palasciano, Maria Ester Modeo, Maria Peragine, Fabrizio Pappagallo, Stefania Pugliese, Carla Di Gennaro (Azienda Ospedaliero-Universitaria Consorziale Policlinico di Bari, Bari, Medicina Interna Ospedaliera "L. D’Agostino", Medicina Interna Universitaria "A. Murri"); Alfredo Postiglione, Maria Rosaria Barbella, Francesco De Stefano (Azienda Ospedaliera Universitaria Policlinico Federico II di Napoli, Medicina Geriatrica Dipartimento di Clinica Medica); Maria Domenica Cappellini, Giovanna Fabio, Sonia Seghezzi, Margherita Migone De Amicis, Marta Mancarella (Fondazione IRCCS Cà Granda Ospedale Maggiore Policlinico, Milano, Unità Operativa Medicina Interna IA); Daniela Mari, Paolo Dionigi Rossi, Sarah Damanti, Barbara Brignolo Ottolini, Giulia Bonini (Fondazione IRCCS Cà Granda Ospedale Maggiore Policlinico, Milano, Geriatria); Gino Roberto Corazza, Emanuela Miceli, Marco Vincenzo Lenti, Donatella Padula (Reparto 11, IRCCS Policlinico San Matteo di Pavia, Pavia, Clinica Medica I); Giovanni Murialdo, Alessio Marra, Federico Cattaneo (IRCS Azienda Ospedaliera Universitaria San Martino-IST di Genova, Genova, Clinica di Medicina Interna 2); Maria Beatrice Secchi, Davide Ghelfi (Ospedale Bassini di Cinisello Balsamo, Milano, Divisione Medicina); Luigi Anastasio, Lucia Sofia, Maria Carbone (Ospedale Civile Jazzolino di Vibo Valentia, Vibo Valentia, Medicina interna); Giovanni Davì, Maria Teresa Guagnano, Simona Sestili (Ospedale Clinicizzato SS. Annunziata, Chieti, Clinica Medica); Gerardo Mancuso, Daniela Calipari, Mosè Bartone (Ospedale Giovanni Paolo II Lamezia Terme, Catanzaro, Unità Operativa Complessa Medicina Interna); Maria Rachele Meroni (Ospedale Luigi Sacco, Milano, Medicina $3^{\circ}$ ); Paolo Cavallo Perin, Bartolomeo Lorenzati, Gabriella Gruden, Graziella Bruno, Cristina Amione, Paolo Fornengo (Dipartimento di Scienze Mediche, Università di Torino, Città della Scienza e della Salute, Torino, Medicina 3); Rodolfo Tassara, Deborah Melis, Lara Rebella (Ospedale San Paolo, Savona, Medicina I); Giuseppe Delitala, Vincenzo Pretti, Maristella Salvatora Masala, Chiara Pes (Ospedale Universitario Policlinico di Sassari, Sassari, Clinica Medica); Luigi Bolondi, Leonardo Rasciti, Ilaria Serio (Policlinico Sant'OrsolaMalpighi, Bologna, Unità Operativa Complessa Medicina Interna); Filippo Rossi Fanelli, Antonio Amoroso, Alessio Molfino, Enrico Petrillo (Policlinico Umberto I, Sapienza Università di Roma, Roma, Medicina Interna $\mathrm{H}$ ); Giuseppe Zuccalà, Francesco Franceschi, Guido De Marco, Cordischi Chiara, Sabbatini Marta, Gabriella D'Aurizio (Policlinico Universitario A. Gemelli, Roma, Roma, Unità Operativa Complessa Medicina d'Urgenza e Pronto Soccorso); Giuseppe Romanelli, Claudia Amolini, Deborah Chiesa, Alessandra Marengoni (Spedali Civili di Brescia, Brescia, Geriatria); Antonio Picardi, Umberto Vespasiani Gentilucci, Paolo Gallo (Università Campus Bio-Medico, Roma, Medicina Clinica-Epatologia);Giorgio Annoni, Maurizio Corsi, Sara Zazzetta, Giuseppe Bellelli, Hajnalka Szabo (Università degli studi di Milano-Bicocca Ospedale S. Gerardo, Monza, Unità Operativa di Geriatria); Franco Arturi, Elena Succurro, Mariangela Rubino, Giorgio Sesti (Università degli Studi Magna Grecia, Policlinico Mater Domini, Catanzaro, Unità Operativa Complessa di Medicina Interna); Paola Loria, Maria Angela Becchi, Gianfranco Martucci, Alessandra Fantuzzi, Mauro Maurantonio (Università di Modena e Reggio Emilia, Medicina MetabolicaNOCSAE, Baggiovara, Modena); Maria Grazia Serra, Maria Antonietta Bleve (Azienda Ospedaliera "Cardinale Panico" Tricase, Lecce, Unità Operativa Complessa Medicina); Laura Gasbarrone, Maria Rosaria Sajeva (Azienda Ospedaliera Ospedale San Camillo Forlanini, Roma, Medicina Interna 1); Antonio Brucato, Silvia Ghidoni, Paola Di Corato (Azienda Ospedaliera Papa Giovanni XXIII, Bergamo, Medicina 1); Giancarlo Agnelli, Emanuela Marchesini (Azienda Ospedaliera Santa Maria della Misericordia, Perugia, Medicina Interna e Cardiovascolare); Fabrizio Fabris, Michela Carlon, Francesca Turatto, Aldo Baritusso, Francesca Turatto, Annalisa Amabile, Elisabetta Omenetto, Paolo Scarinzi (Azienda Ospedaliera Università di Padova, Padova, Clinica Medica I); Roberto 
Manfredini, Christian Molino, Marco Pala, Fabio Fabbian, Benedetta Boari, Alfredo De Giorgi (Azienda Ospedaliera - Universitaria Sant'Anna, Ferrara, Unità Operativa Clinica Medica); Giuseppe Paolisso, Maria Rosaria Rizzo, Maria Teresa Laieta (Azienda Ospedaliera Universitaria della Seconda Università degli Studi di Napoli, Napoli, VI Divisione di Medicina Interna e Malattie Nutrizionali dell'Invecchiamento); Giovanbattista Rini, Pasquale Mansueto, Ilenia Pepe (Azienda Ospedaliera Universitaria Policlinico P. Giaccone di Palermo, Palermo, Medicina Interna e Malattie Metaboliche); Claudio Borghi, Enrico Strocchi, Valeria De Sando, Ilaria Pareo (Azienda Ospedaliera Universitaria Policlinico S. Orsola-Malpighi, Bologna, Unità Operativa di Medicina Interna Borghi); Carlo Sabbà, Francesco Saverio Vella, Patrizia Suppressa, Raffaella Valerio, Pasquale Agosti, Flavia Fontana, Francesca Loparco (Azienda Ospedaliero-Universitaria Consorziale Policlinico di Bari, Bari, Medicina Interna Universitaria C. Frugoni); Stefania Pugliese, Caterina Capobianco (Azienda Ospedaliero-Universitaria Consorziale Policlinico di Bari, Bari, Clinica Medica I Augusto Murri); Luigi Fenoglio, Christian Bracco, Alessia Valentina Giraudo, Elisa Testa, Cristina Serraino (Azienda Sanitaria Ospedaliera Santa Croce e Carle di Cuneo, Cuneo, S. C. Medicina Interna); Silvia Fargion, Paola Bonara, Giulia Periti, Marianna Porzio, Slivia Tiraboschi (Fondazione IRCCS Cà Granda Ospedale Maggiore Policlinico, Milano, Medicina Interna 1B); Flora Peyvandi, Alberto Tedeschi, Raffaella Rossio, Barbara Ferrari (Fondazione IRCCS Cà Granda Ospedale Maggiore Policlinico, Milano, Medicina Interna 2); Valter Monzani, Valeria Savojardo, Christian Folli, Maria Magnini (Fondazione IRCCS Cà Granda Ospedale Maggiore Policlinico, Milano, Medicina Interna Alta Intensità di Cura); Francesco Salerno, Alessio Conca, Giulia Gobbo, Alessio Conca, Giada Pallini, Miriam Valenti (IRCCS Policlinico San Donato e Università di Milano, San Donato Milanese, Medicina Interna); Carlo L. Balduini, Giampiera Bertolino, Stella Provini, Federica Quaglia (IRCCS Policlinico San Matteo di Pavia, Pavia, Clinica Medica III); Franco Dallegri, Luciano Ottonello, Luca Liberale (Università di Genova, Genova, Medicina Interna 1); Wu Sheng Chin, Laura Carassale, Silvia Caporotundo (Ospedale Bassini, Cinisello Balsamo, Milano, Unità Operativa di Geriatria); Giancarlo Traisci, Lucrezia De Feudis, Silvia Di Carlo (Ospedale Civile Santo Spirito di Pescara, Pescara, Medicina Interna 2); Nicola Lucio Liberato, Alberto Buratti, Tiziana Tognin (Azienda Ospedaliera della Provincia di Pavia, Ospedale di Casorate Primo, Pavia, Medicina Interna); Giovanni Battista Bianchi, Sabrina Giaquinto (Ospedale "SS Gerosa e Capitanio" di Lovere, Bergamo, Unità Operativa Complessa di Medicina Generale, Azienda Ospedaliera "Bolognini" di Seriate, Bergamo); Francesco Purrello, Antonino Di Pino, Salvatore Piro (Ospedale Garibaldi Nesima, Catania, Unità Operativa Complessa di Medicina Interna); Renzo Rozzini, Lina Falanga, Elena Spazzini (Ospedale Poliambulanza, Brescia, Medicina Interna e Geriatria); Giuseppe Montrucchio, Elisabetta Greco, Pietro Tizzani, Paolo Petitti (Dipartimento di Scienze Mediche, Università di Torino, Città della Scienza e della Salute, Torino, Medicina Interna 2 U. Indirizzo d'Urgenza); Antonio Perciccante, Alessia Coralli (Ospedale San Giovanni-Decollato-Andisilla, Civita Castellana Medicina); Raffaella Salmi, Piergiorgio Gaudenzi, Susanna Gamberini (Azienda Ospedaliera-Universitaria S. Anna, Ferrara, Unità Operativa di Medicina Ospedaliera II); Andrea Semplicini, Lucia Gottardo (Ospedale SS. Giovanni e Paolo, Venezia, Medicina Interna 1); Gianluigi Vendemiale, Gaetano Serviddio, Roberta Forlano (Ospedali Riuniti di Foggia, Foggia, Medicina Interna Universitaria); Cesare Masala, Antonio Mammarella, Valeria Raparelli (Policlinico Umberto I, Roma, Medicina Interna D); Francesco Violi, Stefania Basili, Ludovica Perri (Policlinico Umberto I, Roma, Prima Clinica Medica); Raffaele Landolfi, Massimo Montalto, Antonio Mirijello, Carla Vallone (Policlinico Universitario A. Gemelli, Roma, Clinica Medica); Martino Bellusci, Donatella Setti, Filippo Pedrazzoli (Presidio Ospedaliero Alto Garda e Ledro,
Ospedale di Arco, Trento, Unità Operativa di Medicina Interna Urgenza/Emergenza); Luigina Guasti, Luana Castiglioni, Andrea Maresca, Alessandro Squizzato, Marta Molaro (Università degli Studi dell'Insubria, Ospedale di Circolo e Fondazione Macchi, Varese, Medicina Interna I); Marco Bertolotti, Chiara Mussi, Maria Vittoria Libbra, Andrea Miceli, Elisa Pellegrini, Lucia Carulli, Francesca Veltri (Università di Modena e Reggio Emilia, AUSL di Modena, Modena, Nuovo Ospedale Civile, Unità Operativa di Geriatria e U.O. di Medicina a indirizzo Metabolico Nutrizionistico); Francesco Perticone, Angela Sciacqua, Michele Quero, Chiara Bagnato, Lidia Colangelo, Tania Falbo (Università Magna Grecia Policlinico Mater Domini, Catanzaro, Unità Operativa Malattie Cardiovascolari Geriatriche); Roberto De Giorgio, Mauro Serra, Valentina Grasso, Eugenio Ruggeri, Benzoni Ilaria (Dipartimento di Scienze Mediche e Chirurgiche, Unità Operativa di Medicina Interna, Università degli Studi di Bologna/Azienda Ospedaliero-Universitaria S.Orsola-Malpighi, Bologna); Andrea Salvi, Roberto Leonardi, Chiara Grassini, Ilenia Mascherona, Giorgio Minelli, Francesca Maltese, Giampaolo Damiani (Spedali Civili di Brescia, U.O. 3 a Medicina Generale); William Capeci, Massimo Mattioli, Giuseppe Pio Martino, Lorenzo Biondi, Monica Ormas, Pietro Pettinari, Roberto Romiti (Clinica Medica, Azienda Ospedaliera Universitaria - Ospedali Riuniti di Ancona); Salvatore Corrao, Silvia Messina, Federica Cavallaro (ARNAS Civico-Di Cristina-Benfratelli - Dipartimento Biomedico di Medicina Interna e Specialistica (Di.Bi.M.I.S.), Palermo); Riccardi Ghio, Serena Favorini, Anna Dal Col (Azienda Ospedaliera Università San Martino, Genova, Medicina III); Salvatore Minisola, Luciano Colangelo (Policlinico Umberto I, Roma, Medicina Interna $F e$ Malattie Metaboliche dell'osso); Antonella Afeltra, Pamela Alemanno, Benedetta Marigliano, Maria Elena Pipita (Policlinico Campus Biomedico Roma, Roma, Medicina Clinica); Pietro Castellino, Julien Blanco, Luca Zanoli (Azienda Ospedaliera Universitaria Policlinico Vittorio Emanuele Ferrarotto, Santa Marta, S. Bambino, Catania, Dipartimento di Medicina); Marco Cattaneo, Paola Fracasso, Maria Valentina Amoruso (Azienda Ospedaliera San Paolo, Milano, Medicina III); Valter Saracco, Marisa Fogliati, Carlo Bussolino (Ospedale Cardinal Massaia Asti, Medicina A); Vittorio Durante, Giovanna Eusebi, Daniela Tirotta (Ospedale di Cattolica, Rimini, Medicina Interna); Francesca Mete, Miriam Gino (Ospedale degli Infermi di Rivoli, Torino, Medicina Interna); Antonio Cittadini, Carlo Vigorito, Michele Arcopinto, Andrea Salzano, Emanuele Bobbio, Alberto Maria Marra, Domenico Sirico (Azienda Policlinico Universitario Federico II di Napoli, Napoli, Medicina Interna e Riabilitazione Cardiologica); Guido Moreo, Francesco Scopelliti, Francesca Gasparini, Melissa Cocca (Clinica San Carlo Casa di Cura Polispecialistica, Paderno Dugnano, Milano, Unità Operativa di Medicina Interna); Alberto Ballestrero, Fabio Ferrando (Clinica Di Medicina Interna ad Indirizzo Oncologico, Azienda Ospedaliera Università San Martino di Genova); Sergio Berra, Simonetta Dassi, Maria Cristina Nava (Medicina Interna, Azienda Ospedaliera Guido Salvini, Garnagnate, Milano); Bruno Graziella, Silvia Ghidoni, Cristina Amione, Stefano Baldassarre, Salvatore Fragapani, Gabriella Gruden (Medicina Interna III, Ospedale S. Giovanni Battista Molinette, Torino); Giorgio Galanti, Gabriele Mascherini, Cristian Petri, Laura Stefani (Agenzia di Medicina dello Sport, AOUC Careggi, Firenze); Margherita Girino, Valeria Piccinelli (Medicina Interna, Ospedale S. Spirito Casale Monferrato, Alessandria); Francesco Nasso, Vincenza Gioffrè, Maria Pasquale (Struttura Operativa Complessa di Medicina Interna, Ospedale Santa Maria degli Ungheresi, Reggio Calabria); Giuseppe Scattolin, Sergio Martinelli, Mauro Turrin (Medicina Interna, Ospedale di Monselice, Padova); Leonardo Sechi, Cristina Catena, Gianluca Colussi (Clinica Medica, Azienda Ospedaliera Universitaria, Udine).

Spanish Hospitals: Ramirez Duque Nieves (Hospital Universitario Virgen del Rocio, Sevilla); Muela Molinero Alberto (Hospital de 
Leon); Abad Requejo Pedro, Lopez Pelaez Vanessa, Tamargo Lara (Hospital del Oriente de Asturias, Arriondas);Corbella Viros Xavier, Formiga Francesc (Hospital Universitario de Bellvitge); Diez Manglano Jesus, Bejarano Tello Esperanza, Del Corral Behamonte Esther, Sevil Puras Maria (Hospital Royo Villanova, Zaragoza); Manuel Romero (Hospital Infanta Elena Huelva); Pinilla Llorente Blanca, Lopez Gonzalez-Cobos Cristina, Villalba Garcia M. Victoria (Hospital Gregorio Marañon Madrid); Lopez Saez, Juan Bosco (Hospital Universitario de Puerto Real, Cadiz); Sanz Baena Susana, Arroyo Gallego Marta (Hospital Del Henares De Coslada, Madrid); Gonzalez Becerra Concepcion, Fernandez Moyano Antonio, Mercedes Gomez Hernandez, Manuel Poyato Borrego (Hospital San Juan De Dios Del Aljarafe, Sevilla); Pacheco Cuadros Raquel, Perez Rojas Florencia, Garcia Olid Beatriz, Carrascosa Garcia Sara (Hospital Virgen De La Torre De Madrid); Gonzalez-Cruz Cervellera Alfonso, Peinado Martinez Marta, Sara Carrascosa Garcia (Hospital General Universitario De Valencia); Ruiz Cantero Alberto, Albarracín Arraigosa Antonio, Godoy Guerrero Montserrat, Barón Ramos Miguel Ángel (Hospital De La Serrania De Ronda); Machin Jose Manuel (Hospital Universitario De Guadalajara); Novo Veleiro Ignacio, Alvela Suarez Lucía (Hospital Universitario De Santiago De Compostela); Lopez Alfonso, Rubal Bran David, Iñiguez Vazquez Iria (Hospital Lucus Augusti De Lugo); Rios Prego Monica (Hospital Universitario De Pontevedra).

\section{Compliance with ethical standards}

Funding The REPOSI study was supported by the Italian Society of Internal Medicine (SIMI), the Ca' Granda Maggiore Policlinico Hospital Foundation, and the Mario Negri Institute of Pharmacological Research. This study was supported by an unrestricted grand from Pfizer to the Scientific Director of Ca' Granda Maggiore Policlinico Hospital Foundation.

Conflicts of Interest Marco Proietti has received a consulting fee from Boehringer Ingelheim. Pier Mannuccio Mannucci has received honoraria for lectures as speaker or chair symposia organized by Bayer, Grifols, Kedrion, LFB, Novo Nordisk and Pfizer, and is a scientific consultant for Bayer, Baxalta and Kedrion. Gregory Y.H. Lip is a consultant for Bayer/Janssen, BMS/Pfizer, Biotronik, Medtronic, Boehringer Ingelheim, Microlife and Daiichi-Sankyo, and a speaker for Bayer, BMS/Pfizer, Medtronic, Boehringer Ingelheim, Microlife, Roche and Daiichi-Sankyo. All other authors have no conflicts of interest to disclose.

\section{References}

1. Mozaffarian D, Benjamin EJ, Go AS, Arnett DK, Blaha MJ, Cushman M, et al. Heart disease and stroke statistics-2016 update: a report from the American Heart Association. Circulation. 2015;133:e38-360.

2. Lip GYH, Lane DA. Stroke prevention in atrial fibrillation. JAMA. 2015;313:1950-62.

3. Freedman B, Potpara TS, Lip GYH. Stroke prevention in atrial fibrillation. Lancet. 2016;388:806-17.

4. Van Gelder IC, Rienstra M, Crijns HJGM, Olshansky B. Rate control in atrial fibrillation. Lancet. 2016;388:818-28.

5. Piccini JP, Fauchier L. Rhythm control in atrial fibrillation. Lancet. 2016;388:829-40.

6. Hohnloser SH, Kuck KH, Lilienthal J. Rhythm or rate control in atrial fibrillation: pharmacological INTERVENTION IN ATRIAL FIBRILLATION (PIAF): a randomised trial. Lancet. 2000;356:1789-94.
7. Smit MD, Crijns HJGM, Tijssen JGP, Hillege HL, Alings M, Tuininga YS, et al. Effect of lenient versus strict rate control on cardiac remodeling in patients with atrial fibrillation data of the RACE II (RAte Control Efficacy in permanent atrial fibrillation II) study. J Am Coll Cardiol. 2011;58:942-9.

8. Wyse DG, Waldo AL, DiMarco JP, Domanski MJ, Rosenberg Y, Schron EB, et al. A comparison of rate control and rhythm control in patients with atrial fibrillation. $\mathrm{N}$ Engl $\mathrm{J}$ Med. 2002;347:1825-33.

9. Roy D, Talajic M, Nattel S, Wyse DG, Dorian P, Lee KL, et al. Rhythm control versus rate control for atrial fibrillation and heart failure. N Engl J Med. 2008;358:2667-77.

10. Noheria A, Shrader P, Piccini JP, Fonarow GC, Kowey PR, Mahaffey $\mathrm{KW}$, et al. Rhythm control versus rate control and clinical outcomes in patients with atrial fibrillation. JACC Clin Electrophysiol. 2016;2:221-9.

11. Ionescu-Ittu R, Abrahamowicz M, Jackevicius CA, Essebag V, Eisenberg MJ, Wynant W, et al. Comparative effectiveness of rhythm control vs rate control drug treatment effect on mortality in patients with atrial fibrillation. Arch Intern Med. 2012;172:997-1004.

12. Nobili A, Licata G, Salerno F, Pasina L, Tettamanti M, Franchi $\mathrm{C}$, et al. Polypharmacy, length of hospital stay, and in-hospital mortality among elderly patients in internal medicine wards. The REPOSI study. Eur J Clin Pharmacol. 2011;67:507-19.

13. Camm AJ, Kirchhof P, Lip GYH, Schotten U, Savelieva I, Ernst $\mathrm{S}$, et al. Guidelines for the management of atrial fibrillation. Eur Heart J. 2010;31:2369-429.

14. Miller MD, Towers A. A manual of guidelines for scoring the Cumulative Illness Rating Scale for geriatrics (CIRS/G). Pittsburg, PA: University of Pittsburg; 1991.

15. Salvi F, Miller MD, Grilli A, Giorgi R, Towers AL, Morichi V, et al. A manual of guidelines to score the modified cumulative illness rating scale and its validation in acute hospitalized elderly patients. J Am Geriatr Soc. 2008;56:1926-31.

16. Katzman R, Brown T, Fuld P, Peck A, Schechter R, Schimmel H. Validation of a short Orientation-Memory-Concentration Test of cognitive impairment. Am J Psychiatry. 1983;140:734-9.

17. Yesavage JA, Brink TL, Rose TL, Lum O, Huang V, Adey M, et al. Development and validation of a geriatric depression screening scale: a preliminary report. J Psychiatr Res. 1982;17:37-49.

18. Mahoney FI, Barthel DW. Functional evaluation: the Barthel Index. Md State Med J. 1965;14:61-5.

19. Martin-Doyle W, Essebag V, Zimetbaum P, Reynolds MR. Trends in US hospitalization rates and rhythm control therapies following publication of the AFFIRM and RACE trials. J Cardiovasc Electrophysiol. 2011;22:548-53.

20. Hanon O, Vidal JS, Pisica-Donose G, Benattar-Zibi L, Bertin P, Berrut G, et al. Therapeutic management in ambulatory elderly patients with atrial fibrillation: the S.AGES cohort. J Nutr Health Aging. 2015;19:219-27.

21. Wutzler A, von Ulmenstein S, Attanasio P, Huemer M, Parwani AS, Boldt L-H, et al. Treatment of nonagenarians with atrial fibrillation: insights from the Berlin atrial fibrillation (BAF) registry. J Am Med Dir Assoc. 2015;16:969-72.

22. Kirchhof P, Benussi S, Kotecha D, Ahlsson A, Atar D, Casadei B, et al. 2016 ESC guidelines for the management of atrial fibrillation developed in collaboration with EACTS. Europace. 2016;18:1609-78.

23. Shariff N, Desai RV, Patel K, Ahmed MI, Fonarow GC, Rich MW, et al. Rate-control versus rhythm-control strategies and outcomes in septuagenarians with atrial fibrillation. Am J Med. 2013;126:887-93. 
24. Kumana CR, Cheung BMY, Cheung GTY, Ovedal T, Pederson B, Lauder IJ. Rhythm vs. rate control of atrial fibrillation metaanalysed by number needed to treat. $\mathrm{Br} \mathrm{J}$ Clin Pharmacol. 2005;60:347-54.

25. Ha ACT, Breithardt G, Camm AJ, Crijns HJ, Fitzmaurice GM, Kowey PR, et al. Health-related quality of life in patients with atrial fibrillation treated with rhythm control versus rate control: insights from a prospective international registry (registry on cardiac rhythm disorders assessing the control of atrial fibrillation: RECORD-AF). Circ Cardiovasc Qual Outcomes. 2014;7:896-904.

26. Perez A, Touchette DR, DiDomenico RJ, Stamos TD, Walton SM. Comparison of rate control versus rhythm control for management of atrial fibrillation in patients with coexisting heart failure: a cost-effectiveness analysis. Pharmacotherapy. 2011;31:552-65.
27. Friberg L, Tabrizi F, Englund A, Wyse D, Waldo A, DiMarco J, et al. Catheter ablation for atrial fibrillation is associated with lower incidence of stroke and death: data from Swedish health registries. Eur Heart J. 2016;37:1825-33.

28. Purmah Y, Proietti M, Laroche C, Mazurek M, Tahmatzidis D, Boriani G, et al. Rate vs. rhythm control and adverse outcomes among European patients with atrial fibrillation. Europace. 2018;20(2):243-52.

29. Tsadok MA, Jackevicius CA, Essebag V, Eisenberg MJ, Rahme $\mathrm{E}$, Humphries $\mathrm{KH}$, et al. Rhythm versus rate control therapy and subsequent stroke or transient ischemic attack in patients with atrial fibrillation. Circulation. 2012;126:2680-7.

30. Vidaillet HJ. Rate control vs. rhythm control in the management of atrial fibrillation in elderly persons. Am J Geriatr Cardiol. $2005 ; 14: 73-80$. 